Reporte de Caso/ Case Report

\title{
Meningioma de nervio óptico y seno cavernoso: Reporte de caso
}

\author{
*Jenny Ku-Lozano ${ }^{1}$, Margarita Samudio², Vanessa Luján-Donayre ${ }^{1,3}$, Doris Quiroz-Cerna ${ }^{1,3}$ \\ ${ }^{1}$ Hospital Almenara, Departamento de Oftalmología. Lima, Perú \\ ${ }^{2}$ Universidad Nacional de Asunción, Instituto de Investigaciones en Ciencias de la Salud. Paraguay \\ ${ }^{3}$ Mácula D\&T, Lima, Perú
} Cómo referenciar este artículo/
How to reference this article:
Ku-Lozano J, Samudio M., Luján-Donayre V, Quiroz-Cerna D. Meningioma de nervio óptico y seno cavernoso: Reporte de caso. Mem. Inst. Investig. Cienc. Salud. 2019; 17(2): 107-111

\section{RE S U M E N}

Los meningiomas de nervio óptico y de seno cavernoso son patologías poco frecuentes, y hasta el momento no ha habido ningún reporte de que se presenten ambos en un mismo paciente. Cabe resaltar que cuando llega un paciente a consulta diagnosticado con alguna patología, asumimos que este diagnóstico es adecuado y pertinente. Pero en nuestro caso, el paciente presentó signos y síntomas de etiología desconocida que hicieron que se re-evaluarán los diagnósticos oftalmológicos que traía la paciente, encontrando que había sido tratada por un diagnóstico que no le correspondía y a su vez este hallazgo nos ayudó a encontrar la verdadera causa.

Palabras clave: nervio optico, seno cavernoso, meningioma.

\section{Optic nerve and cavernous sinus meningiomas: case report}

\begin{abstract}
A B S T R A C T
Optic nerve and cavernous sinus meningiomas are uncommon pathologies, and so far there have not been previously reported to occur in the same patient. It should be emphasized that when a patient arrives at a doctor's office diagnosed with pathology, we assume that this diagnosis is appropriate and pertinent. But in our case, the patient presented signs and symptoms of unknown etiology that led to a re-evaluation of the previous ophthalmological diagnoses that the patient brought, finding that she had been treated for a diagnosis that did not match with all her clinical sign and symptoms and this helped us to find the real cause.
\end{abstract}

Keywords: optic nerve, cavernous sinus, meningioma.

\section{INTRODUCCIÓN}

Los meningiomas son la causa principal de los tumores primarios de cerebro, representando el $14-18 \%$ del total de tumores intracraneales ${ }^{(1)}$ y su incidencia varía de 1-8.4 por cada 100000 personas; aumentando progresivamente en cada década de la vida ${ }^{(2)}$.

El meningioma de nervio óptico es poco frecuente (1-2\% del total), de crecimiento lento, extraaxial ${ }^{(3)}$, con predominio del sexo femenino, siendo la edad media 45 años $y$, si aparece en niños puede estar relacionada con la neurofibromatosis tipo $2^{(4)}$.

Eventualmente, puede invadir la órbita y/o extenderse intracranealmente, infiltrando el quiasma óptico, el nervio óptico contralateral, la carótida interna, el seno

Fecha de recepción: abril 2019. Fecha de aceptación: junio 2019

*Autor correspondiente: Jenny Ku-Lozano. Hospital Almenara, Departamento de Oftalmología. Lima, Perú

Email:kamlin22@gmail.com 
cavernoso, el ligamento falciforme, las apófisis clinoides anteriores, el área frontal, el bulbo olfatorio y la región selar. ${ }^{(5)}$

Su clínica involucra una pérdida de la visión lentamente progresiva y variable $(97 \%)$, asociada a diplopía, ptosis y limitaciones de los movimientos oculares debido al efecto compresivo de la masa, dependiendo de la extensión y el sitio de afección ${ }^{(6,7)}$

En cuanto al meningioma de seno cavernoso y parasillares, su frecuencia es de $17 \%{ }^{(8)}$ y puede asociarse a cefaleas ocasionales, dolores trigeminales, signo de Horner, oftalmoplejía con parálisis de los pares craneales (III, IV y/o VI), hipoestesia del territorio del nervio trigémino, exoftalmia por compresión de las venas oftálmicas y ptosis (en $50 \%$ de los casos). ${ }^{(9)}$

Al ser estas patologías poco frecuentes, es común el diagnóstico erróneo, y al tener consecuencias permanentes en la agudeza visual, es importante detectarlas a tiempo.

Asimismo, en Perú no se han reportado casos similares de presentación en un solo paciente, razones por las que se realiza el presente reporte.

\section{Presentación del caso}

Paciente de 53 años, sexo femenino, procedente de Lima (Perú), con antecedente de Glaucoma desde hace 3 años, en tratamiento. Acude a la consulta por presentar diplopía vertical y horizontal de 6 días de evolución.

Añadió que había sido atendida en un centro oftalmológico particular, donde se le realizaron exámenes serológicos (hemograma, glucosa, urea, creatinina) e imagenológicos (RMN, TAC y Angioresonancia de cerebro) con resultados normales y recibió tratamiento con corticoides por 4 días, lo cual resolvió parcialmente el cuadro.

Al examen oftalmológico presentó agudeza visual (con corrección) de 20/20 y 20/25 en ojos derecho e izquierdo respectivamente; presiones intraoculares de 16 $\mathrm{mmHg}$ en ambos ojos. Pupilas isocóricas, fotorreactivas. Biomicroscopía: sin alteraciones en ambos ojos.

Fondo de ojo derecho: dentro de los límites normales, con nervio óptico rosado, relación copa/disco $(R C D)$ : 0.3 , mácula y vascularización sin alteraciones (Figura $1 \mathrm{~A}$ ). Fondo de ojo izquierdo: nervio óptico rosado, RCD: 0.5. Mácula y vascularización sin alteraciones (Figura 1B).

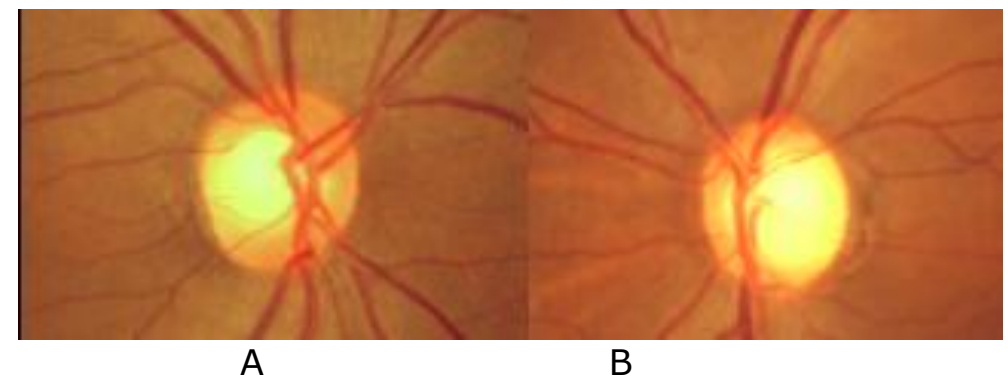

Figura 1: Fondos de ojo derecho e izquierdo, respectivamente.

Siguiendo con el examen, se valoró la motilidad ocular con las diferentes posiciones de la mirada, encontrándose una paresia del III par craneal izquierdo (Figura 2).

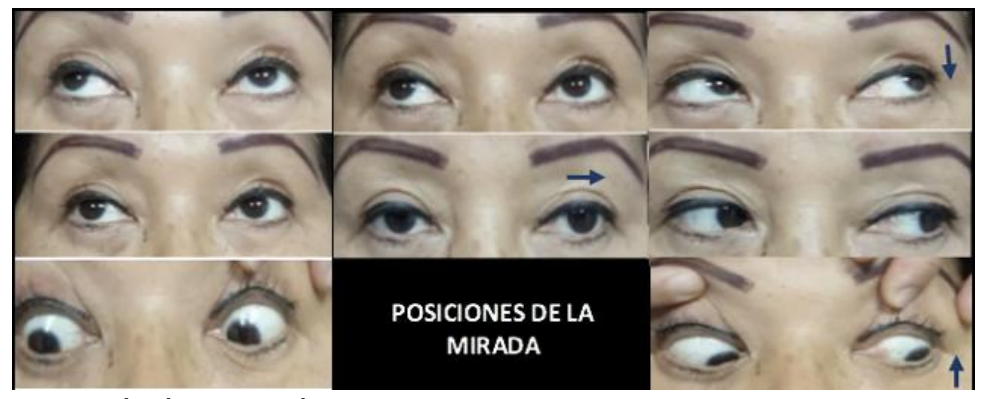

Figura 2: Posiciones de la mirada. 
Ante los hallazgos, se decide realizar algunos estudios para definir la patología ante la cual nos encontrábamos.

Se realiza un campo visual (Figura 3), una tomografía axial computarizada de nervio óptico con valoración de capa de células ganglionares (Figura 4), observando imágenes no compatibles con el diagnóstico de glaucoma.

Se decide entonces realizar una Tomografía Axial computarizada (Figura 5) y una Resonancia magnética de órbita.
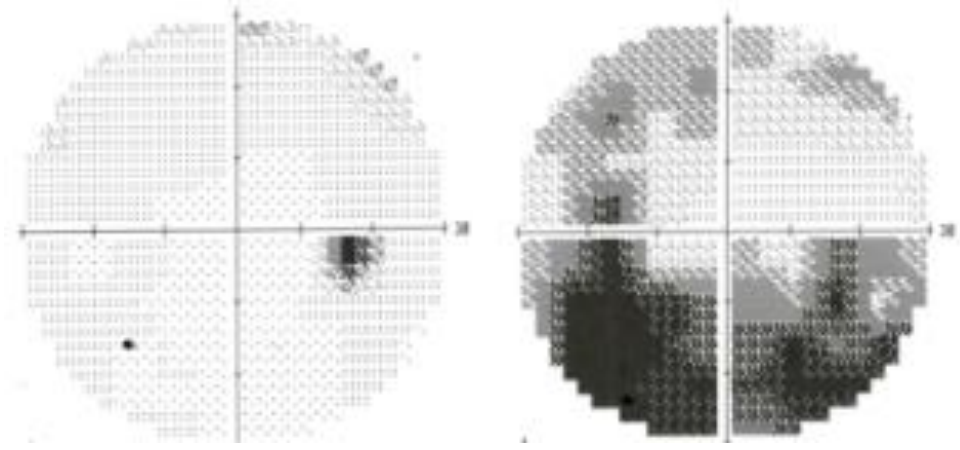

Figura 3: Campo visual de ambos ojos

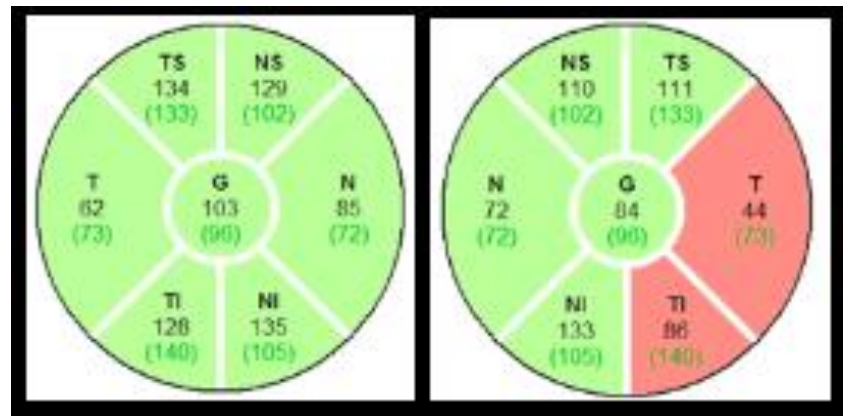

Figura 4: Capa de células ganglionares de ambos ojos

Se observó presencia de calcificaciones, tortuosidad y engrosamiento en el trayecto del nervio óptico que se proyectaba hasta la llegada al seno cavernoso, lo cual era compatible con un meningioma del nervio óptico y del seno cavernoso y nos explicaba la clínica (Figura 5).

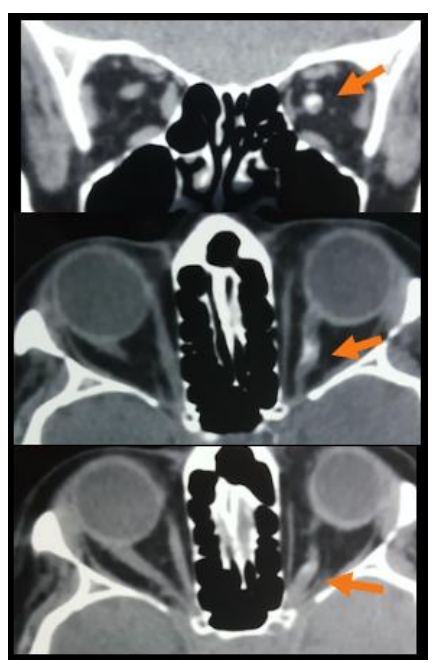

Figura 5: Tomografía axial computarizada de ambas órbitas

Con estos hallazgos, se decide continuar tratamiento con corticoides por 3 semanas más y uso de prismas con control semanal. Al mes y ante la remisión del cuadro, se 
retira el tratamiento corticoide y prismas, quedando en observación periódica. Cabe resaltar que hasta la fecha no se ha evidenciado recurrencia.

\section{DISCUSIÓN}

Los meningiomas de nervio óptico y del seno cavernoso, son patologías benignas poco frecuentes ${ }^{(1-4)}$, que suponen el $1 \%$ de todos los tumores de la órbita y $1 / 3$ de los tumores primarios del nervio óptico ${ }^{(10)}$.

Éstos pueden producir función anómala de los sistemas visuales tanto aferente como eferente ${ }^{(11)}$ y manifestaciones clínicas variables, dependiendo de la extensión y el sitio de afección ${ }^{(6,7)}$.

Su diagnóstico es a menudo un reto para el médico, ya sea por su naturaleza de crecimiento lento, pérdida de la agudeza visual insidiosa o apariencia del nervio óptico variable (disco normal, atrófico o edematoso) hace que sean diagnosticados equívocamente ${ }^{(12)}$.

Generalmente son confundidos con casos de papilitis, atrofia optica o neuritis óptica isquémica $(48 \%)^{(2)}$, incluso con glaucoma, como fue en nuestro caso.

El tiempo de demora para llegar al diagnóstico adecuado fue casi 4 años en nuestro estudio, lo que coincide con los resultados encontrados por Kahraman, donde el tiempo medio de demora fue de 62.60 meses ( 5 años $)^{(12)}$.

Así también, encontraron que el error diagnóstico más frecuente fue la falla en un correcto examen clínico $(60 \%)$, lo que generó una hipótesis incorrecta, solicitud de exámenes auxiliares diagnósticos no relevantes y por ende tratamientos inadecuados ${ }^{(2,13)}$.

Por otro lado, Kahraman mencionó que también se solicitaron algunos exámenes pertinentes, pero el resultado salió dentro de los valores normales (como en el caso de nuestra paciente); evidenciándose que éstos fueron leídos incorrectamente por un tecnólogo no radiólogo $(45.5 \%)$ y $54.5 \%$ fueron realizados incorrectamente (sin secuencia orbital o contraste, según el caso) $)^{(13)}$.

En cuanto al tratamiento; éste es controversial; pero siguiendo las pautas propuestas por Berman y Miller, nuestra paciente calificaba para mantener un manejo conservador, con controles 2 veces al año y realización de exámenes auxiliares anuales ${ }^{(6,14)}$.

El pronóstico visual es variable, dependiendo fundamentalmente de la agudeza visual inicial, la detección y el tratamiento oportuno ${ }^{(12-16)}$.

De otra manera, el pronóstico es pobre, como evidenciaron Kahraman en su estudio, donde 16 de 25 pacientes estudiados (64\%) tuvieron repercusión visual ${ }^{(13)}$ y Dutton, quien reportó que el $85 \%$ del total de pacientes demostraron una disminución de la visión ${ }^{(16)}$.

Los tumores del nervio óptico y del seno cavernoso son poco frecuentes, de evolución lenta y su diagnóstico es generalmente de exclusión.

Los diagnósticos preestablecidos sesgados, el examen clínico oftalmológico inadecuado, la solicitud de los exámenes auxiliares (RMN cerebral / órbitas con contraste) y la interpretación errónea de los mismos, fueron la causa principal de errores diagnósticos y retraso en el correcto; con peores resultados visuales y mayor costo (Más visitas y pruebas).

El principal objetivo debe enfocarse en realizar una exhaustiva historia clínica, solicitar los exámenes auxiliares pertinentes para poder realizar un adecuado y oportuno diagnóstico, el cual nos permita asegurar un resultado visual favorable, establecer el control local del tumor y reducir al mínimo los riesgos de morbilidad relacionada con el tratamiento.

Aspectos éticos: El presente reporte de caso contó con el consentimiento informado de la paciente para la presentación de la información contenida en el artículo.

Conflictos de intereses: No existen conflictos de interés asociados a la materia del artículo. 


\section{REFERENCIAS BIBLIOGRÁFICAS}

1. Nakamura M, Roser F, Michel J, Jacobs C, Samii M, De Tribolet $N$, et al. The natural history of incidental meningiomas. Neurosurgery. 2003;53(1): 62-71.

2. Jung-Feng Mao, Xiao-Bo Xia, Xiang-Bo Tang, Xue-Yong Zhang DW. Analyses on the misdiagnoses of 25 patients with unilateral optic nerve sheath meningioma. Int J Ophthalmol2016;9(9):1315-9.

3. Muñoz L, Muci-Mendoza R, Karam E. Meningiomas de la vaina del nervio óptico. Análisis de una experiencia de 27 años en la unidad de Neurooftalmología del Hospital Vargas de Caracas. Gac Méd Caracas. 2011; 119(1):48-54

4. Miller N. Clinical Neuro-Ophthalmology. 6th ed. Walsh and Hoyts. Ed. Philadelphia, Pennsylvania: Lippincott Williams \& Wilkins; 2005.

5. Brell $M$, Villa $S$, Teixidor $P$, Lucas $A$, Ferrán $\mathrm{E}$, Marín $\mathrm{S}$. Fractionated stereotactic radiotherapy in the treatment of exclusive cavernous sinus meningioma: Functional outcome, local control, and tolerance. Surg Neurol. 2006;65(1): 28-33.

6. Shapey J, Sabin H, Danesh-Meyer HV, et al. Diagnosis and management of optic nerve sheath meningiomas. J Clin Neurosci 2013; 20(8): 1045-1056.

7. Nidamanuri $P$, Shastin D, Nannapaneni R. Cavernous sinus meningioma presenting as third nerve palsy in pregnancy. BMJ Journals; case reports (2018)

8. Al-Okaili R, Krejza F, Wang S, Woo J, Melhem E. Advanced MR Imaging
Techniques in the Diagnosis of Intraaxial Brain Tumors in Adults. Radio Graphics 2006;26:173-189

9. Louis M, Goga D, François P, Laure B. Ptosis révélant un méningiome du sinus caverneux. Journal Français d'Ophtalmologie;2013;36(10):197200.

10. Gold D, Lewis R. Oftalmología. American Medical Association. Ed. Marbán S.L. España, 2006

11. Mallory K, Draper $E$, Maglione A, Seidler K. Progressive sixth nerve palsy secondary to intracavernous arachnoid cyst and complicated by contralateral optic nerve sheath meningioma. European Journal of Ophthalmology. 2019, 6.

12. Baehring JM. Tram track sign. J Neurooncol. 2007;85(1):75.

13. Kahraman-Koytak P, Bruce B, Peragallo J, Newman N, Biousse V. Diagnostic Errors in Initial Misdiagnosis of Optic Nerve Sheath Meningiomas. JAMA Neurol., 2019; 76(3):326-332.

14. Berman D, Miller. New concepts in the management of optic nerve sheath meningiomas. Ann Acad Med Singapore. 2006;35:168-174.

15. Saeed P, Rootman J, Nugent R, White $\mathrm{V}$, Mackenzie I, Koornneef L. Optic nerve sheath meningiomas. Ophthalmology. 2003;110(10):20192030.

16. Parker $\mathrm{R}$, Ovens $\mathrm{C}$, Fraser C, Samarawickrama C. Optic nerve sheath meningiomas: prevalence, impact and managemente strategies. Eye and Brain 2018:10 85-99. 